Letter to the Editor

\title{
A de novo monoallelic CTLA-4 deletion causing pediatric onset CVID with recurrent autoimmune cytopenias and severe enteropathy
}

A R T I C L E I N F O

\section{Keywords:}

CTLA-4

B cells

T cells

Hypogammaglobulinemia

Enteropathy

Autoimmune cytopenias

\section{To the editor}

Monoallelic mutations in CTLA4 have been recently described in a small number of individuals with a phenotype of common variable immunodeficiency (CVID). The clinical features are variable and include hypogammaglobulinemia, autoimmune cytopenias, lymphoproliferative disease, and autoimmune enteropathy $[1,2]$. More recently, a large cohort study of 133 patients with CTLA-4 haploinsufficiency highlighted the susceptibility of these patients to infections with cytomegalovirus and Epstein Barr virus [3]. The majority of CTLA4 haploinsufficiency is caused by nonsense, missense, or frameshift mutations [3]. Only two patients diagnosed with CVID in adulthood have been reported to carry a large mono-allelic heterozygous deletion spanning the 2q33.2-2q33.3 region, which includes CTLA4, CD28 and ICOS [3]. We now report a female patient with a de novo monoallelic deletion in CTLA4 presenting with pediatric-onset CVID complicated with severe enteropathy and recurrent autoimune cytopenias.

The proband was born to non-consanguineous parents of Italian descent with no family history of immunodeficiency or immune dysregulation. The proband presented with recurrent respiratory infections from the age of two years. At three and seven years of age, she presented with immune thrombocytopenic purpura (ITP) that responded well to treatment with high-dose intravenous immunoglobulin (IVIG). At eight years of age, the patient presented with her first episode of Coombs positive autoimmune hemolytic anemia (AIHA). Immunological evaluation at this time revealed pan-hypogammaglobulinemia (IgG $449 \mathrm{mg} / \mathrm{dl}$, normal 633-1016; IgA: < $5 \mathrm{mg} / \mathrm{dl}$, normal 41-315; IgM:42 mg/dl, normal 56-261) leading to a diagnosis of CVID. The patient was started on IVIG replacement therapy and on prophylaxis with amoxicillin and cotrimoxazole. The patient had no evidence of infections with CMV, EBV or hepatitis. Due to refractory AIHA and ITP, the patient also received steroids and anti-CD20 treatment. At 14 years of age, immunological evaluation showed reduction of naïve $\mathrm{T}$ cells and recent thymic emigrants, expansion of $\mathrm{CD}^{+}$and $\mathrm{CD}^{+}$effector memory $\mathrm{T}$ cells and $\mathrm{B}$ cell maturational arrest at the $\mathrm{IgM}^{+}$ memory stage (Supplemental Table 1). At this time, she developed chronic diarrhea. Histological examination revealed increased $\mathrm{T}$ cell infiltrate in the duodenum (Fig. 1A and B) and increased glandular apoptotic bodies with an inflammatory cell infiltrate in the colonic lamina propria (Fig. 1C and D). Treatment with oral budesonide resulted in partial improvement. TNF inhibition with infliximab was initiated, but was stopped due to development of transaminitis. The patient was then put on adalimumab and azathioprine with partial clinical response. At 15 years of age, lung CT scan showed nodular lesions with ground glass abnormalities and opacification (Fig. 1E). The patient is currently 24 years old with a height and weight below the third percentile.

Targeted next generation sequencing (NGS) of the proband for 264 genes associated with primary immunodeficiency revealed a monoallelic deletion of exons 1-4 of CTLA4, which was not found in either parent (Fig. 1F). CGH array (180 K Agilent Technologies) confirmed the monoallelic deletion of 2q33.2-2q33.3 previously reported in two patients with adult-onset CVID, which also includes CTLA4, ICOS, and CD28 [3]. Flow cytometric evaluation of CTLA-4 expression showed severely decreased protein expression in activated $\mathrm{CD} 4^{+} \mathrm{T}$ cells and an almost complete lack of Foxp3 $+\mathrm{T}$ regulatory cells (Tregs) (Fig. 1G). (CTLA-4 MFI: HD 16,5; Pt:9,77) (percentages of CTLA-4+Foxp3+ CD4 + T cells (\%) HD: 3,6; Pt: 0,18). One of the previously reported patients had arthritis, viral, and fungal infections, while the other developed AIHA and CVID [3]. In contrast, our proband presented with very early-onset respiratory infections at the age of two years, followed by the development of autoimmune cytopenias and hypogammaglobulinemia in childhood. Enviromental exposures, modifier genes, and/ or epigenetic modifications may be responsible for the differences observed in these patients. Of note, the index patient developed severe B cell lymphopenia over time (Supplemental Table 1), as previously described in a subset of CTLA-4 haploinsufficient patients [3].

Here, we report a pediatric-onset CVID patient harbouring a de novo 2q33.2-2q33.3 monoallelic deletion leading to nearly absent CTLA4 expression. This is the third patient worldwide harbouring this deletion, and the second one with a de novo deletion. The patient's phenotype resulted particularly complex and resembles the previously described presentation in CTLA-4 haploinsufficient patients [3], although in a more severe form and with an early-onset. Considering the complexity of this disorder, further longitudinal studies are warranted in order to better define the appropriate therapeutic approach for affected patients. 
A.

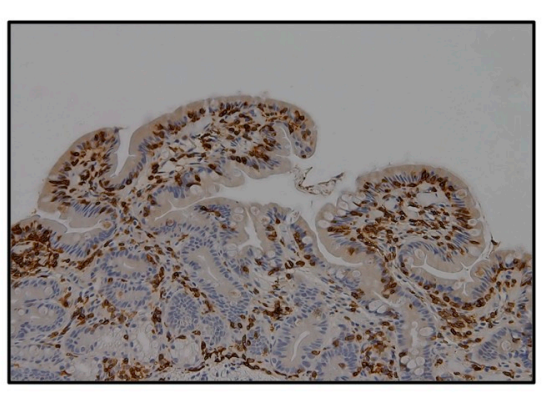

C.

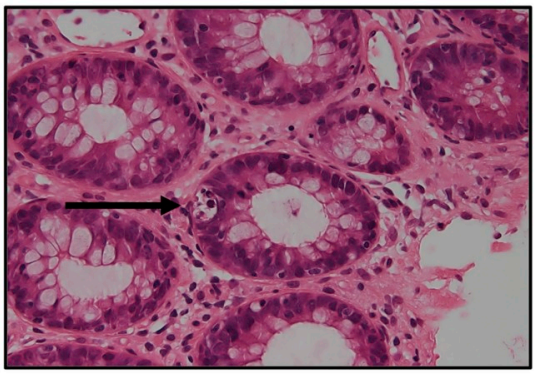

F.
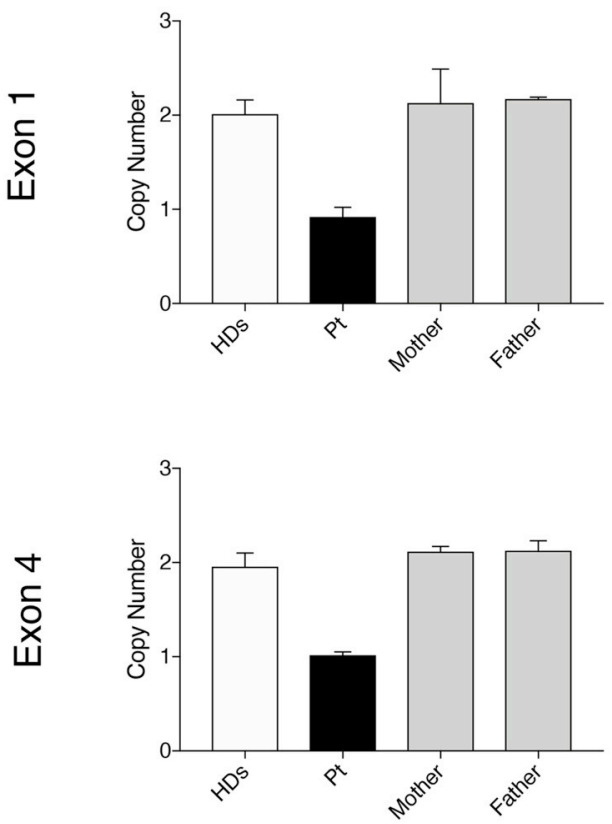

B.

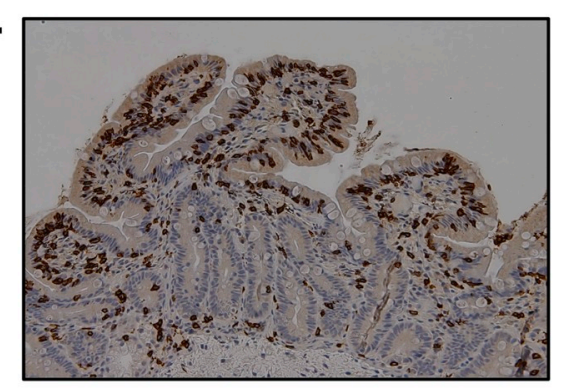

D.

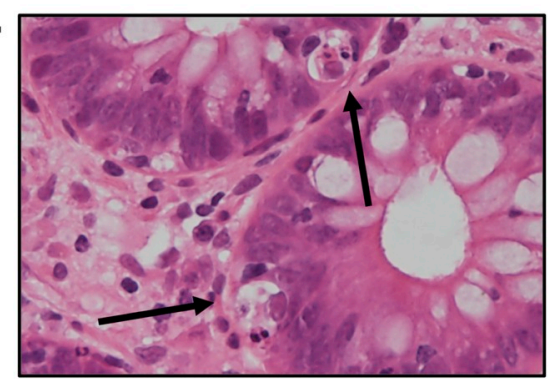

E.
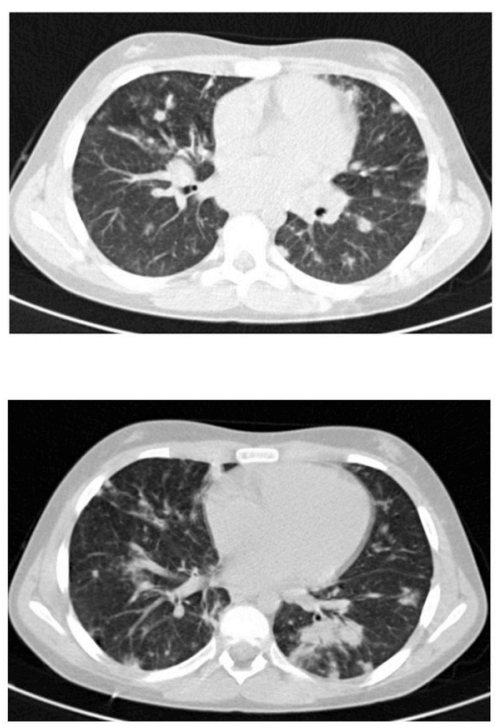

G.

NS

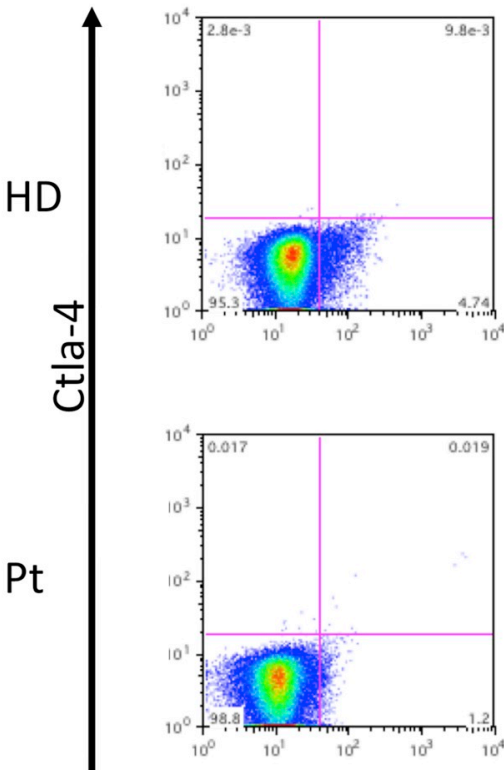

Stim.
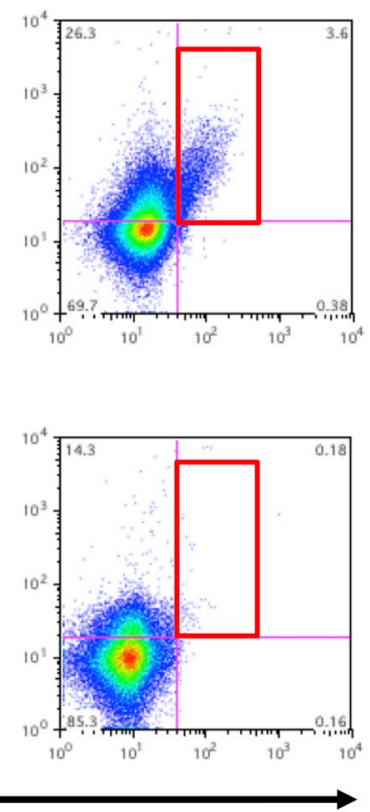

Foxp3

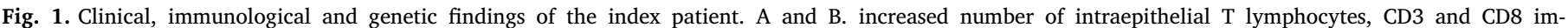

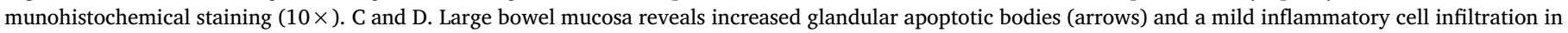

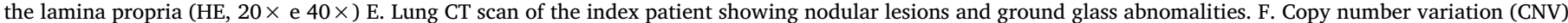

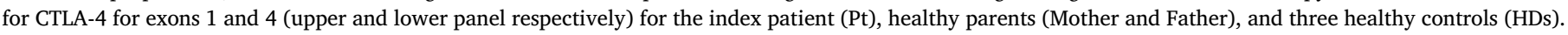

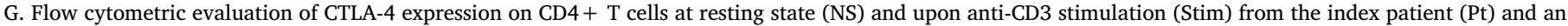
age-matched healthy control (HD).

Supplementary data to this article can be found online at https:// doi.org/10.1016/j.clim.2018.10.007.

\section{Funding}

The research leading to these results has received funding from the European Community's Seventh Framework Programme FP7/
2007-2013 under grant agreement no 201549 (EURO-PADnet HEALTH-F2-2008-201549) and from the Italian Ministerial Grant GR2010-2315762. The research leading to these results also received funding from the "Fondazione C. Golgi", Brescia, Italy and the Jeffrey Modell Foundation. 


\section{Conflict of interest}

The authors declare no conflict of interest.

\section{Acknowledgements}

We thank the patient, the patients' family and the nurses for all their efforts, and Drs. Raif Geha and Janet Chou for the next-generation sequencing results.

\section{References}

[1] D. Schubert, C. Bode, R. Kenefeck, T.Z. Hou, J.B. Wing, A. Kennedy, A. Bulashevska, B.S. Petersen, A.A. Schäffer, B.A. Grüning, S. Unger, N. Frede, U. Baumann, T. Witte, R.E. Schmidt, G. Dueckers, T. Niehues, S. Seneviratne, M. Kanariou, C. Speckmann, S. Ehl, A. Rensing-Ehl, K. Warnatz, M. Rakhmanov, R. Thimme, P. Hasselblatt, F. Emmerich, T. Cathomen, R. Backofen, P. Fisch, M. Seidl, A. May, A. SchmittGraeff, S. Ikemizu, U. Salzer, A. Franke, S. Sakaguchi, Walker LSK, D.M. Sansom, Grimbacher B. Autosomal dominant immune dysregulation in humans with CTLA4 mutations. Nat. Med. 20 (12) (2014 Dec) 1410-1416, https://doi.org/10.1038/nm. 3746.

[2] H.S. Kuehn, W. Ouyang, B. Lo, E.K. Deenick, J.E. Niemela, D.T. Avery, J.N. Schickel, D.Q. Tran, J. Stoddard, Y. Zhang, D.M. Frucht, B. Dumitriu, P. Scheinberg, L.R. Folio, C.A. Frein, S. Price, C. Koh, T. Heller, C.M. Seroogy, A. Huttenlocher, V.K. Rao, H.C. Su, D. Kleiner, L.D. Notarangelo, Y. Rampertaap, K.N. Olivier, J. McElwee, J. Hughes, S. Pittaluga, J.B. Oliveira, E. Meffre, T.A. Fleisher, S.M. Holland, M.J. Lenardo, S.G. Tangye, Uzel G. Immune dysregulation in human subjects with heterozygous germline mutations in CTLA-4, Science 345 (6204) (2014 Sep 26) 1623-1627, https://doi.org/10.1126/science.1255904.

[3] C. Schwab, A. Gabrysch, P. Olbrich, V. Patiño, K. Warnatz, D. Wolff, A. Hoshino, M. Kobayashi, K. Imai, M. Takagi, I. Dybedal, J.A. Haddock, D. Sansom, J.M. Lucena, M. Seidl, A. Schmitt-Gräff, V. Reiser, F. Emmerich, N. Frede, A. Bulashevska,
U. Salzer, D. Schubert, S. Hayakawa, S. Okada, M. Kanariou, Z.Y. Kucuk, H. Chapdelaine, L. Petruzelkova, Z. Sumnik, A. Sediva, M. Slatter, P.D. Arkwright, A. Cant, H.M. Lorenz, T. Giese, V. Lougaris, A. Plebani, C. Price, K.E. Sullivan, M. Moutschen, J. Litzman, T. Freiberger, F.L. van de Veerdonk, M. Recher, M.H. Albert, F. Hauck, S. Seneviratne, J.P. Schmid, A. Kolios, G. Unglik, C. Klemann, C. Speckmann, S. Ehl, A. Leichtner, R. Blumberg, A. Franke, S. Snapper, S. Zeissig, C. Cunningham-Rundles, L. Giulino-Roth, O. Elemento, G. Dückers, T. Niehues, E. Fronkova, V. Kanderová, C.D. Platt, J. Chou, T. Chatila, R. Geha, E. McDermott,

S. Bunn, M. Kurzai, A. Schulz, L. Alsina, F. Casals, A. Deyà-Martinez, S. Hambleton, H. Kanegane, K. Taskén, O. Neth, B. Grimbacher, Phenotype, penetrance, and treatment of 133 CTLA-4-insufficient individuals, J. Allergy Clin. Immunol. 18 (2018 May 3) 30630-30634, https://doi.org/10.1016/j.jaci.2018.02.055.

Vassilios Lougaris $^{\mathrm{a}, *}$, Manuela Baronio ${ }^{\mathrm{a}}$, Luisa Gazzurelli ${ }^{\mathrm{a}}$, Tiziana Lorenzini ${ }^{\mathrm{a}}$, Maurizio Fuoti ${ }^{\mathrm{b}}$, Daniele Moratto ${ }^{\mathrm{c}}$, Anna Bozzola ${ }^{\mathrm{d}}$, Chiara Ricci ${ }^{\mathrm{e}}$, Maria Pia Bondioni ${ }^{\mathrm{f}}$, Alberto Ravelli ${ }^{\mathrm{b}}$, Vincenzo Villanacci ${ }^{\mathrm{d}}$, Alessandro Plebani ${ }^{\mathrm{a}}$

a Pediatrics Clinic and Institute for Molecular Medicine A. Nocivelli, Department of Clinical and Experimental Sciences, University of Brescia, ASST-Spedali Civili of Brescia, Brescia, Italy

${ }^{\mathrm{b}}$ Pediatrics Clinic, ASST-Spedali Civili of Brescia, Brescia, Italy ${ }^{\mathrm{c}}$ Institute for Molecular Medicine A. Nocivelli, Department of Pathology, Laboratory of Genetic Disorders of Childhood, Department of Molecular and Translational Medicine, University of Brescia, Spedali Civili di Brescia, Italy

${ }^{\mathrm{d}}$ Institute of Pathology, ASST-Spedali Civili of Brescia, Brescia, Italy

${ }^{\mathrm{e}}$ Gastroenterology Unit, Department of Clinical and Experimental Sciences, University of Brescia, Viale Europa 11, 25123 Brescia, Italy ${ }^{\mathbf{f}}$ Pediatric Radiology, University of Brescia, ASST Spedali Civili di Brescia, Brescia, Italy E-mail address: vlougarisbs@yahoo.com (V. Lougaris)

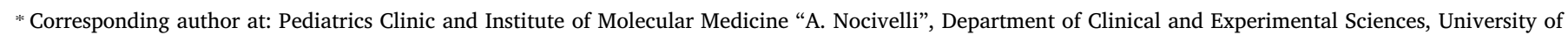
Brescia, ASST-Spedali Civili of Brescia, Piazzale Spedali Civili 1, 25123 Brescia, Italy. 